\title{
VIRTUAL SCREENING STUDIES OF TWO CLOSELY RELATED WITHANOLIDES TO CONTROL CELL PROLIFERATION AND INDUCTION OF CELL SENESCENCE
}

\author{
S. Rashmi ${ }^{1}$, S. Nivethitha ${ }^{1}$, C. N. Hemalatha ${ }^{2}$ and M. Vijey Aanandhi*3 \\ ${ }^{1}$ Department of pharmaceutical chemistry, School of Pharmaceutical Sciences, Vels University \\ (VISTAS), Chennai-600117, Tamil Nadu, India \\ ${ }^{2}$ Research Scholar, Department of Pharmaceutical Chemistry, Vels University (VISTAS), \\ Chennai-600117, Tamil Nadu, India \\ ${ }^{3}$ Department of Pharmaceutical Chemistry and Analysis, School of Pharmaceutical Sciences, \\ Vels University (VISTAS), Chennai-600117,Tamil Nadu, India \\ *E-mail: hodpchemistry@velsuniv.ac.in
}

\begin{abstract}
Withania somnifera, a reputed herb which is also known as Ashwagandha of the family solanaceae or nightshade family. It comprises a large number of steroidal lactones known as Withanolides which show various pharmacological activities. Withania exhibits anti-tumor, anti-inflammatory,immunomodulatory and anti-antigenic properties.To control of cell proliferation and stress resulting in induction of cellular senescence it involves proteins and hence were considered as the major tool in the present study. The objective of this study was to study the binding energy of Withania somnifera biological active compounds, and drug likeliness by insilico techniques for anticancer activity. The proteins were retrieved from PDB bank and plant data compounds are taken from a literature survey and the active constituents are alkaloids (isopellertierine, anferine), steroidal lactones (Withanolides, Withaferin A), saponins containing an additional Acyl group (Sitoindoside VII and VIII), and Withanoloides. The active constituents are docked by using AutoDock 4.2 Software with the 4 PDB IDs such as 3N8E, 3D09, 2FLE and 1AXC. From the docking results, Withanolides showing satisfactory dock score values. These compounds are visualized by using Discovery studio 4.1 Visualizer followed by DruLiTo software which satisfies the Lipinski's properties for all the compounds. The compounds have been showed good interactions and binding energy with the proteins.
\end{abstract}

Keywords: Withania somnifera, Withaferin A, Withanolides, cellular senescence

@ RASĀYAN. All rights reserved

\section{INTRODUCTION}

Ashwagandha (Withania somnifera: solanaceae), A traditional system of medicine in India for about thousands of years and are popularly called as an adaptogenic herb. They are abundantly found in Asian countries like India, Pakistan, and Afghanistan. W.somniferais known among scholars as Indian ginseng or winter cherry which possess various pharmacological activities. Its mechanisms such as antiinflammatory, anti-cancer, anti-diabetic, anti-stress, antioxidant, neuroprotective and immuno modulatory potentials were demonstrated in very few studies based on cell and animal models ${ }^{1-4}$.other investigators indicated that leaf extracts of W.somnifera also has anti-bacterial property ${ }^{5}$. Steroidal alkaloids, saponins, and steroidal lactones are the major constituents of extracts, obtained from various parts of ashwagandha. Steroidal lactones are the class of chemicals collectively known as Withanolides (ergostane skeleton) ${ }^{6}$. Withanolides consists of six-membered lactone ring with C28 steroidal nucleus with an aC9 side chain. So far 12 alkaloids, 35 Withanolides and several Sitoindosides have been isolated and their structures have been elucidated $^{7,8}$. Withanine, Somniferine, Somnine, somniferinine, Withanine, pseudo-Withanine, Tropine, Pseudotropine, Cuscohygrine, Isopelletierine, Anaferine, and Anahydrine are the major alkaloids present in them. The saponins such as Sitoindoside VII and Sitoindoside VIII, are also present in the roots of Withania somnifera. Pharmacological activities involve activation of immune cells mainly 
lymphocytes and phagocytes, involves in potent antioxidant effects, generally it promotes wellness by reducing the effects of stress ${ }^{9}$.

Cancer is one of the major diseases and challenging to the medicinal system to produce potent and the site-specific anti-cancer drugs. Rich source of bioactive compounds have played a significant role in modern days particularly in the medicinal plants and serves as an important target for the discovery of new drugs. WI-A include inhibition of protein kinase $\mathrm{C}^{10}{ }^{11}$, inhibition of AKt and Raf-1 pathways resulting in tumor suppression by induction of apoptosis and cell adhesion.

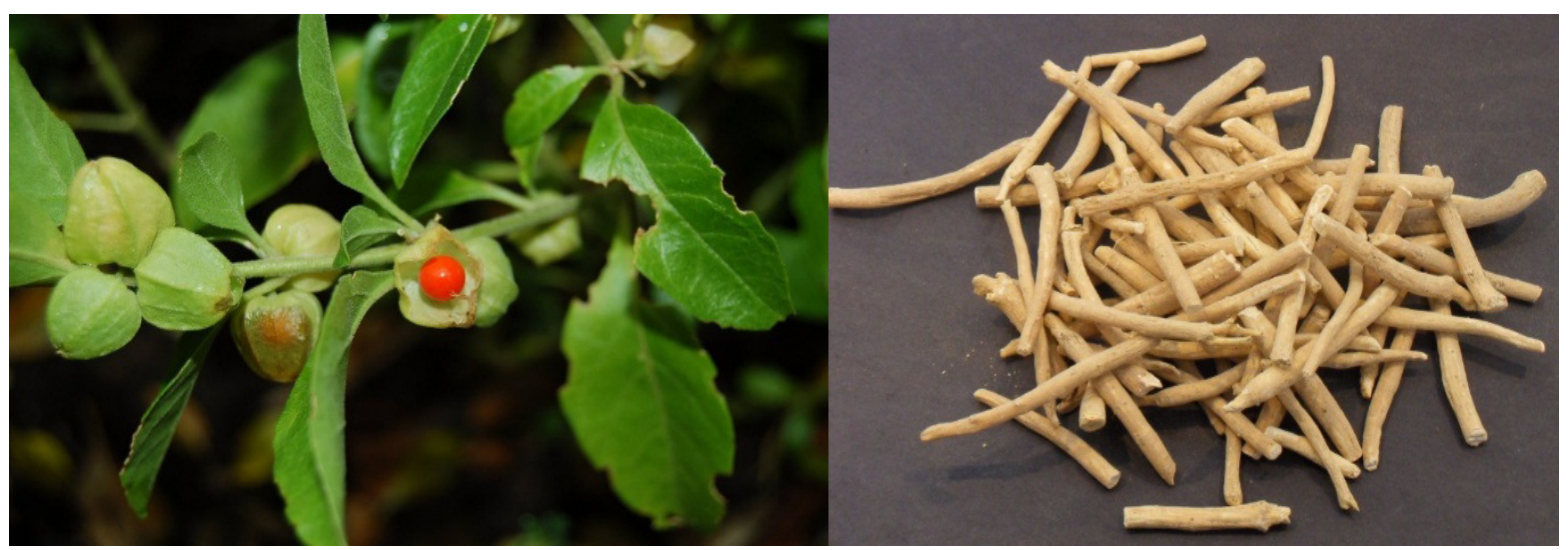

Fig.-1: Withania somnifera

\section{EXPERIMENTAL}

Withania somnifera derived compounds: compounds selected for this study are Withaferin-A, WithaferinA-diacetate and Withanone and their structures are shown in Table-1. Lipinski's properties such as molecular weight, log p, molar refractivity, number of hydrogen bond acceptors and donors taken from SCFBio software for $W$.somnifera derived plant compounds and they satisfy Lipinski's rule of five for Drug-Likeness. The values of the Lipinski's properties are highlighted in Table-2.

Table-1: Compounds and their Structures

WITHAFERIN-A $\quad$ WITHAFERIN-A-DIACETATE


RASĀYAN $J$. Chem.

Vol. 11 | No. 1 |339 - 344 | January - March | 2018

Table-2: Lipinski’s Rule

\begin{tabular}{c|c|c|c}
\hline COMPOUNDS & WITHAFERIN-A & $\begin{array}{c}\text { WITHAFERIN-A- } \\
\text { DIACETATE }\end{array}$ & WITHANONE \\
\hline MOL.WT & 470.0000 & 554.0000 & 312.0000 \\
\hline Log $\mathrm{p}$ & 3.352900 & 4.49450 & 3.39530 \\
\hline H bond acceptor & 6 & 8 & 6 \\
\hline H bond donor & 2 & 0 & 5 \\
\hline Molar refractivity & 124.46355 & 143.558075 & 77.145782 \\
\hline
\end{tabular}

\section{Protein preparation}

The proteins with three-dimensional structures were downloaded from the RCSB protein data bank (PDBID: 3N8E, 3D09, 2FLU and 1AXC) and used for docking studies. Further, polar hydrogen atoms were added and water molecules present in them were removed.Kollman united atom partial charges were also assigned. The PDBQT files that contain proteins are used to execute Autodock.

\section{Protein-ligand preparation}

The roots and expansion were identified from the three-dimensional structures of proteins as required by the docking programs. The torsion angles were identified for three compounds taken for study, were five for Withaferin-A, seven for Withaferin-A-diacetate and four for Withanone compound that denotes the flexibility of the ligand molecule.

\section{Ligand docking}

The receptor (macromolecule) and the ligand molecule interactions were carried out using AutoDock tool.The molecular docking logs and their analysis were analyzed using the graphical user interface of ADT using AutoDock 4.2 tool. Finally, the final docking results were noted at the end of the docking process (for each of the four protein)in order to confirm the accuracy of the maximum binding energy, inhibitory constant, hydrogen bond interactions and ligand deficiency. The dock score values are tabulated in Table-3.

Table-3: Chemical Compounds and Their Dock Score Values

\begin{tabular}{|c|c|c|c|}
\hline Compound & Protein & Binding Energy & Hydrogen Bond Contacts \\
\hline \multirow{4}{*}{ WITHAFERIN-A } & $1 \mathrm{AXC}$ & -6.96 & $\begin{array}{c}\text { 5: Tyr (151) N...H } \\
\operatorname{Arg}(156) \mathrm{O} . . \mathrm{H}\end{array}$ \\
\hline & 2fle & -6.73 & $\begin{array}{c}\text { 3:Tyr }(103) \mathrm{H} \ldots . . \mathrm{H} \\
\text { Gln }(104) \mathrm{H} \ldots \mathrm{N}\end{array}$ \\
\hline & 3D09 & -7.23 & $\begin{array}{c}\text { 3: Thr (102) H...H } \\
\text { Phe (113) H...N } \\
\text { Asn (268) H...D21 }\end{array}$ \\
\hline & $3 \mathrm{~N} 8 \mathrm{E}$ & -8.59 & 1: $\operatorname{Arg}(513) \mathrm{NH} 1 \ldots \mathrm{H}$ \\
\hline \multirow{4}{*}{$\begin{array}{l}\text { WITHAFERIN-A } \\
\text { DIACETATE }\end{array}$} & $1 \mathrm{AXC}$ & -6.51 & 1: Arg (146) H...H \\
\hline & 2fle & -8.49 & 1: Asn (83) H...N \\
\hline & 3D09 & -6.81 & $\begin{array}{c}\text { 2: } \operatorname{Arg}(202) H . . . H 22 \\
\text { His (233) H...D1 }\end{array}$ \\
\hline & $3 \mathrm{~N} 8 \mathrm{E}$ & -6.87 & 1: Leu (450) H...N \\
\hline \multirow{4}{*}{ WITHANONE } & $1 \mathrm{AXC}$ & -7.11 & 3: Gly (142) H...N \\
\hline & 2fle & -7.15 & $\begin{array}{l}\text { 2: Lys (70) H...N } \\
\text { Ile (72) H...N }\end{array}$ \\
\hline & 3D09 & -6.92 & 3: Tyr (205) H...N \\
\hline & $3 \mathrm{~N} 8 \mathrm{E}$ & -6.65 & $\begin{array}{c}\text { 3: } \operatorname{Arg}(513) H . . . H 22 \\
\text { His (590) H...D1 }\end{array}$ \\
\hline
\end{tabular}


RASĀYAN $J$. Chem.

Vol. 11 | No. 1 |339 - 344 | January - March | 2018

Table-4: Visualization of the Docked Compounds

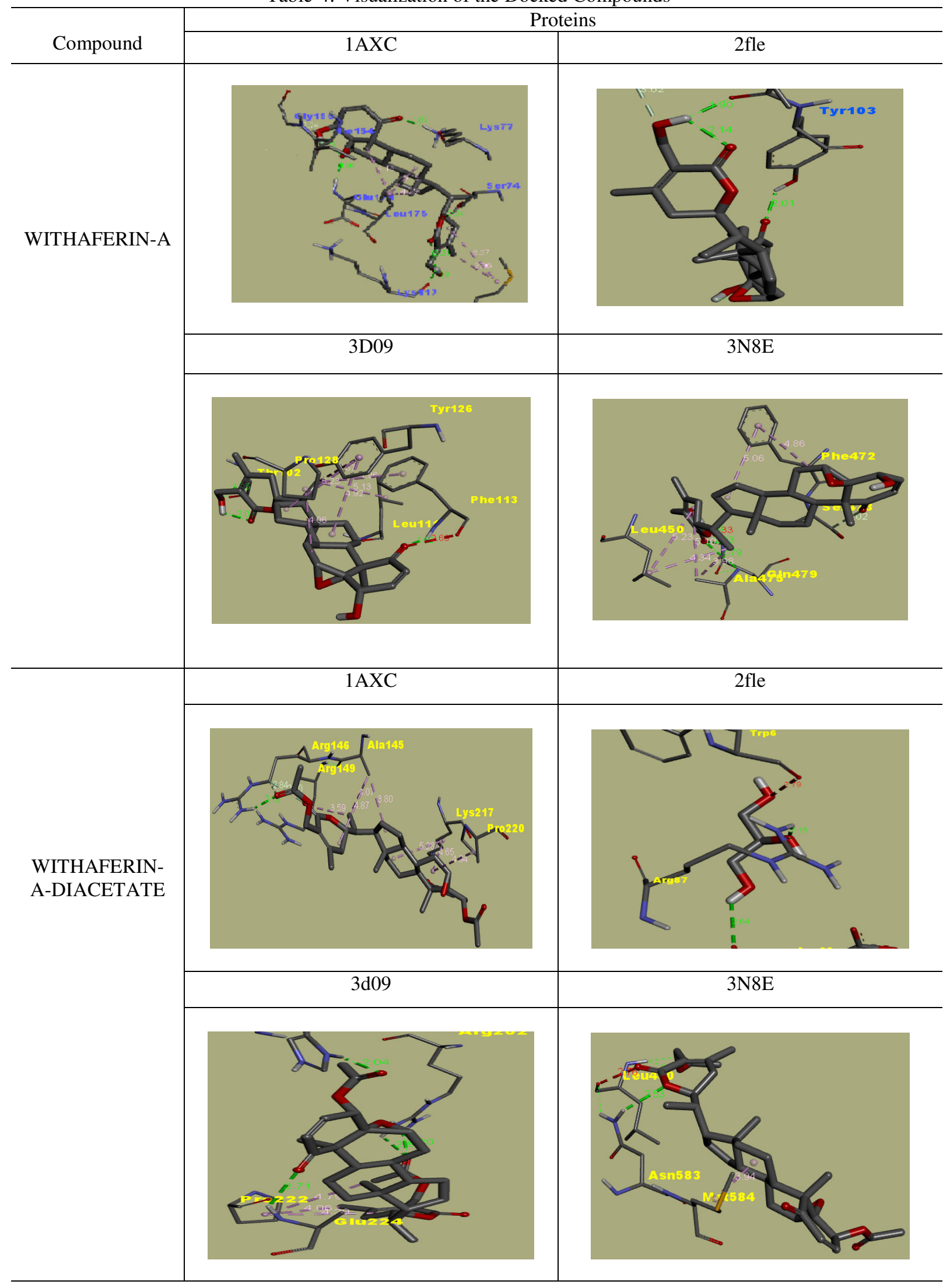


RASĀYAN $J$. Chem.

Vol. 11 | No. 1 |339 - 344 | January - March | 2018

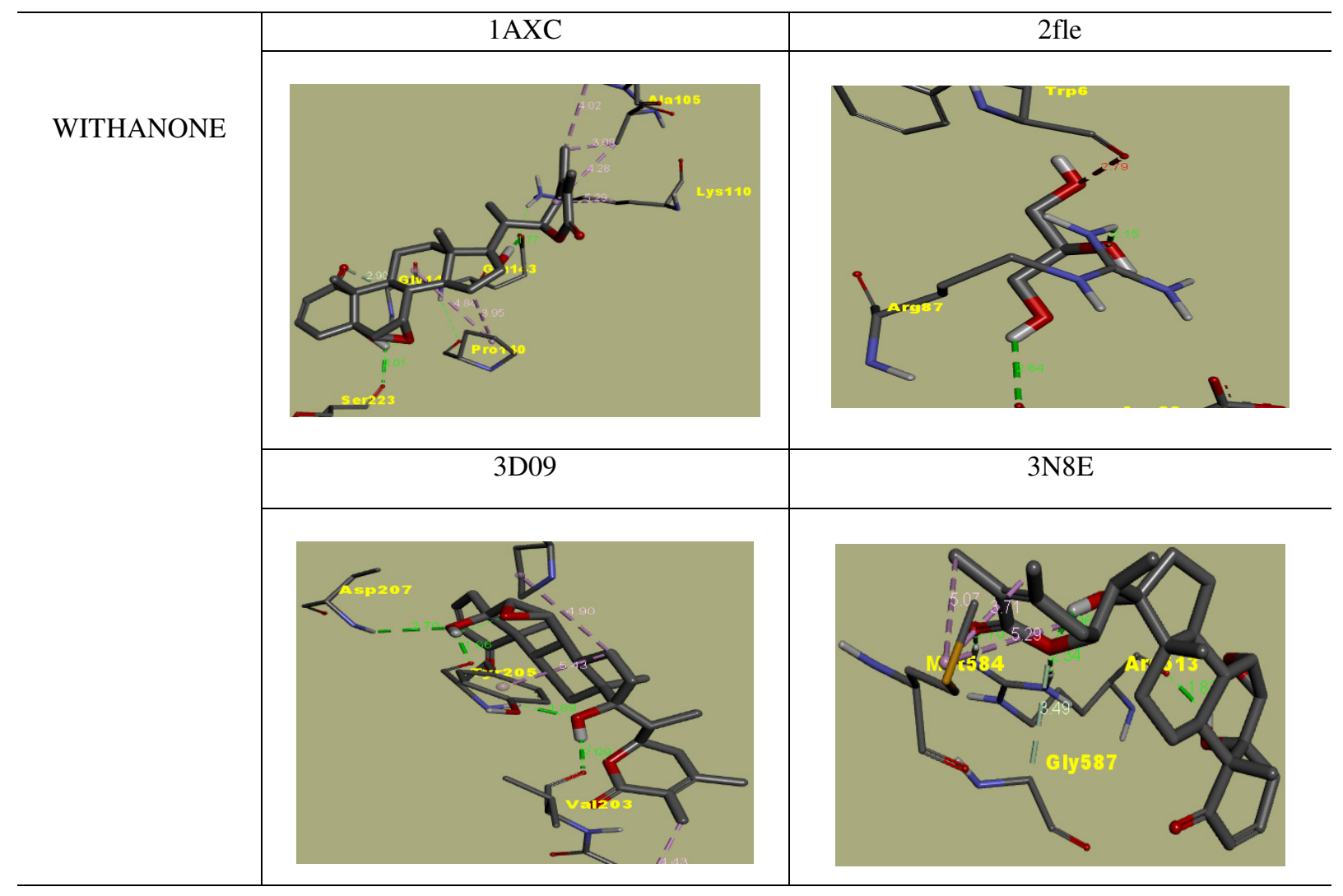

\section{Visualization}

The small and macromolecule applications of proteins involves the usage of Discovery studio 4.1 visualizer; a free, molecular modeling environment tool. It is developed by Accelrys which specializes in scientific software products. Its usage is most relevant to pharmaceutical and biotechnology industries and they are also used in wide range of academic and commercial entities. The visualized and the docked compounds are tabulated in Table-4.

\section{RESULTS AND DISCUSSION}

The pharmacological activities of the medicinal plant, Withania somniferaare adaptogenic, antiinflammatory, anti-cancer and anti-oxidant effects. In this study, we focused on the anti-cancer activity of the plant. The compounds such as Withaferin-A, Withanone, and Withaferin-A-diacetate showed better binding features with targets. Amino acid residues such as arginine, asparagine, tyrosine, histidine, and leucine have bound to the above-mentioned compounds. The compounds showed good binding energy thus these compounds can be effectively used for the treating anti-cancer activity.

\section{CONCLUSION}

The docking study has been carried out to find the binding energy of plant chemical constituents present in Withania somniferamainly in three compounds such as Withaferin-A, Withaferin-A-diacetate, and Withanone. From the RCSB protein data bank, four relevant proteins were selected for the docking study. We interpret the results and studied the binding energy and hydrogen bond contacts which are efficient for anti-cancer therapy.

Among the chemical compounds and proteins with their binding energies, we intended that Withaferin- A has more potent effects than the other constituents present in the plant. Withaferin, Withaferin A diacetate and Withanoneof Withania somnifera medicinal plant has been showing good interaction with their respective targets so these plant constituents will have a more potent effect on treating cancer. In future 


\section{RASĀYAN J. Chem.}

Vol. 11 | No. 1 |339 - 344 | January - March | 2018

studies on the synthesis of these compounds and their effectiveness in the wet lab will confirm the present findings.

\section{REFERENCES}

1. R. Aalinkeel, Z. Hu, B. B. Nair, D. E. Sykes, J. L. Reynolds, Evid. Based Complement Alternat Med., 7, 177(2010).

2. N. Shah, H. Kataria, S.C. Kaul, T. Ishii, G. Kaur, Cancer Sci., 100, 1740(2009).

3. E. Mayola Gallerne, D.D. Esposti, C. Martel, S. Pervaiz, Apoptosis, 16, 1014(2011).

4. H. Nakajima, Y. Wakabayashi, K. Wakamatsu, G. Imokawa, Phytother. Res., 25(9),1398(2011).

5. L. Davis and G. Kuttan, J. Ethnopharmacol., 71(1-2), 193(2000).

6. S.K. Kulkarni, A. Dhir, Prog. Neuropsychopharmacol Biol. Psychiatry., 32, 1093(2008).

7. L.C. Mishra, B.B. Singh, S. Dagenais, Altern. Med. Rev., 5, 334(2000).

8. H. Matsuda, T. Murakami, A. Kishi, M. Yoshikawa, Bioorg. Med. Chem., 9, 1499(2001).

9. Dhananjay Dwivedi, Mayuri Thanwar et al., Rasayan Journal of Chemistry, 8, 522(2015).

10. J.D. Adam Jr., J. Yang, L.C. Mishra, B.B. Singh, Altern. Ther. Health Med., 8, 18(2002).

11. N. Sen, B. Banerjee, B.B. Das, A. Ganguly, T. Sen, Cell Death Differ, 14, 358(2007).

[RJC-1848/2017] 